\title{
HOMEOMORPHISMS OF A SURFACE WHICH ACT TRIVIALLY ON HOMOLOGY
}

\author{
DENNIS L. JOHNSON
}

\begin{abstract}
Let $\mathfrak{T}$ be the mapping class group of a surface of genus $\boldsymbol{g}>\mathbf{3}$, and $G$ the subgroup of those classes acting trivially on homology. An infinite set of generators for $\mathcal{G}$, involving three conjugacy classes, was obtained by Powell. In this paper we improve Powell's result to show that $G$ is generated by a single conjugacy class and that $[\mathscr{R}, \mathscr{G}]=\mathscr{G}$.
\end{abstract}

I. Let $M=M_{g, 1}$ be an orientable surface of genus $g \geqslant 3$ with one boundary component. (We shall frequently refer to the boundary curve as "the hole".) Let $\Re=\Re_{g, 1}$ be its mapping class group (that is, homeomorphisms of $M$ which are 1 on the boundary modulo homeomorphisms which are isotopic to 1 by an isotopy which is fixed on the boundary), and let $g=g_{g, 1}$ be the mapping classes of $\mathbb{N}$ which induce the identity map on the homology group $H_{1}(M, Z)$. The group $g$ is of specific interest to topologists for a number of reasons. For example, every hommology 3-sphere is obtained as a Heegaard decomposition with glueing map in 9 ; more precise knowledge about 9 could thus conceivably give some information about homology spheres. For the group-theoretically inclined, 9 supports a number of interesting problems. For example, it is an open question as to whether it is finitely generated. At present, information concerning $G$ is scarce; the main references are given at the end.

Let $\alpha$ be any bounding simple closed curve (BSCC) in $M$. It has then a well defined genus $g(\alpha)$, namely, the genus of the surface it bounds. (In contrast to the case of a closed surface, $\alpha$ bounds only on one side; the other side contains the hole.) Consider the group $\mathscr{T}_{k} \subset g$ generated by all twists $T_{\alpha}$ on BSCC's $\alpha$ of genus $k ; \mathscr{T}_{k}$ is clearly a normal subgroup of $\mathscr{N}$, since the genus of $\alpha$ is invariant under any homeomorphism $h$ of $M$, and $T_{h(\alpha)}=h T_{\alpha} h^{-1}$.

If $\alpha_{1}, \alpha_{2}$ are two disjoint, homologous SCC's with $\alpha_{i}$ not homologous to zero (we shall write " $\simeq$ " for "homologous") then $\left(\alpha_{1}, \alpha_{2}\right)$ also has a genus $g\left(\alpha_{1}, \alpha_{2}\right)$, since $\alpha_{1}, \alpha_{2}$ bound a piece of $M$. If we let $\mho_{k}$ be the group generated by all maps of the form $T_{\alpha_{1}} T_{\alpha_{2}}{ }^{-1}$ with $g\left(\alpha_{1}, \alpha_{2}\right)=k$ then $\mathscr{W}_{k} \subset g$ is also normal in $\mathfrak{T}$. We shall speak of such a map as "a generator of $\mathscr{W}_{k}$ "; likewise, if $\alpha$ is a BSCC and $g(\alpha)=k, T_{\alpha}$ will be called "a generator of $\mathscr{T}_{k}$ ". Note that all generators of a given type are conjugate in $\mathfrak{N}$; this is just the same as saying, for example, that if $\alpha, \beta$ are BSCC's of the same genus, then

Received by the editors April 13, 1978 and, in revised form, July 25, 1978.

AMS (MOS) subject classifications (1970). Primary 57A05, 55A99. 
there is a homeomorphism $h$ such that $\beta=h(\alpha)$.

Jerry Powell has shown in [P] that, for a closed surface of genus $g>3, g_{g}$ is generated by the generators of $\mathcal{T}_{1}, \mathcal{T}_{2}$ and $\mathcal{W}_{1}$, that is, $\mathscr{G}_{g}=\mathscr{W}_{1} \cdot \mathcal{T}_{1} \cdot \mathscr{T}_{2}$. Here $g_{g}$ is the closed surface version of $g_{g, 1}$, while $\mathcal{T}_{k}$ has generators $T_{\alpha}$ with $\alpha$ bounding genus $k$ on one side or the other (thus $\mathscr{T}_{k}=\mathscr{T}_{g-k}$ for a closed surface), and likewise for $\mho_{k}$. We shall use Powell's result to produce a stronger one, namely that $G=W_{1}$, this result holding for both closed surfaces and surfaces with one hole. We will then use this to show that $[\Re, g]=9$.

For $g=\$, 2$ the above results fail. The group $\Phi_{1}$ is trivial (this is because $\pi_{1}\left(M_{1}\right)$ is abelian) and a theorem of Nielsen (see [MKS, Theorem 3.9, p. 165]) implies that $g_{1,1}$ is infinite cyclic and generated by a twist on the boundary. For a closed surface of genus 2, $\mho_{1}$ and $\sigma_{2}$ are clearly trivial and Powell shows that $g=\mathscr{T}_{1}$. Using the methods of this paper, Powell's result also implies fairly easily that $g_{2,1}=\mathscr{W}_{1} \cdot \mathscr{T}_{1}$ and $\mathscr{T}_{2} \subset \mathcal{W}_{1}$. We also find that $[\Re, g] \neq G$ for $g=2$. The author has shown by different methods (unpublished) that $I_{2} /\left[\mathfrak{T}_{2}, g_{2}\right]$ (which must be cyclic) has $Z_{10}$ as a quotient and that the corresponding group for $g_{2,1}$ has $Z \oplus Z_{2}$ as a quotient. In the remainder of the paper we will assume $g \geqslant 3$.

II. If $\Re_{g, 1}$ is the mapping class group of a surface with one hole and $\mathfrak{N}_{g}$ that of a closed surface, there is a natural surjection $\mathfrak{N}_{g, 1} \stackrel{p}{\rightarrow} \mathfrak{R}_{g}$ obtained by "filling in the hole". The kernel of $p$ is generated by "moving the hole around"; precisely, by:

(a) twisting the hole,

(b) maps of type $T_{\alpha_{1}} T_{\alpha_{2}}{ }^{-1}$ with $\alpha_{1}, \alpha_{2}$ disjoint, homologous, and $g\left(\alpha_{1}, \alpha_{2}\right)=$ $g-1$ : see Figure 1. The map illustrated there has the effect of sliding the hole around the second handle. Note that twisting the hole itself is just $T_{\alpha}$ for $g(\alpha)=g$. Also, these maps are all in $g_{g, 1}$; hence we get an exact sequence: $0 \rightarrow \operatorname{Ker} p \rightarrow g_{g, 1} \rightarrow g_{g} \rightarrow 0$.

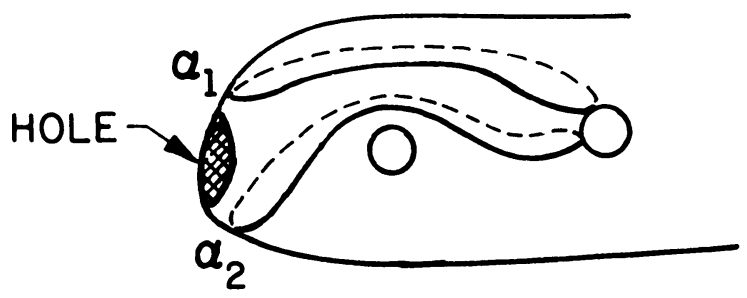

FIGURE 1

Now Powell's theorem says that $g_{g}=\mathscr{W}_{1} \cdot \sigma_{1} \cdot \mathscr{T}_{2}$. But a $\mathscr{W}_{1}$ generator for a closed surface is $T_{\alpha_{1}} T_{\alpha_{2}}{ }^{-1}$, where $\alpha_{1}, \alpha_{2}$ bound a surface of genus 1 on one

'I am particularly indebted to Joan Birman for many interesting discussions concerning these matters and for encouraging this work. 
side and $g-2$ on the other. Suppose $D \subset M_{g}$ is a disc, and so its complement is $M_{g, 1}$. We may change the $\alpha$ 's by an isotopy so that they are disjoint from the disc $D$, and hence $T_{\alpha_{1}} T_{\alpha_{2}}{ }^{-1}$ also defines a map in $\Phi_{g, 1}$ lifting that in $g_{g}$. Its genus is either 1 or $g-2$, depending on the position of the disc $D$ with respect to $\alpha_{1}, \alpha_{2}$. Likewise, $\mathcal{T}_{1}$ generators of the closed surface may be lifted to $\mathscr{T}_{1}$ or $\mathscr{T}_{g-1}$ generators in $\mathscr{G}_{g, 1}$, and $\mathcal{T}_{2}$ to $\mathscr{T}_{2}$ or $\mathcal{T}_{g-2}$. Since these lifted generators plus the generators of $\operatorname{Ker} p$ generate $g_{g, 1}$ we see that $g_{g, 1}$ is generated by the $\mathscr{W}_{k}$ 's and $\mathscr{T}_{k}$ 's. Our program will be to show that all of the $\mathscr{T}_{k}$ 's are contained in $\mathscr{T}_{1} \cdot \mathscr{T}_{2}$, and that $\mathscr{T}_{1}, \mathcal{T}_{2}$ and the $\mathscr{W}_{k}$ 's are all contained in $\mathscr{W}_{1}$. This result will also clearly hold for $g_{g}$ as well.

III. First we show that $\mathscr{W}_{k} \subset \mathcal{W}_{1}$. Consider Figure 2: the genus is $>3$, and $g\left(\alpha_{1}, \alpha_{2}\right)=g\left(\alpha_{2}, \alpha_{3}\right)=1, g\left(\alpha_{1}, \alpha_{3}\right)=2$. Hence $T_{\alpha_{1}} T_{\alpha_{2}}{ }^{-1}$ and $T_{\alpha_{2}} T_{\alpha_{3}}{ }^{-1}$ are in $\mathcal{W}_{1}$, and their product $T_{\alpha_{1}} T_{\alpha_{3}}{ }^{-1}$ is also; but the latter is a typical generator of $\mathcal{W}_{2}$. Since all these generators are conjugate in $\Re$, and $\mathscr{W}_{1}$ is normal, we get $\mathscr{V} \int_{2} \subset \mathcal{W}_{1}$. By induction (extending the genus of the surface between $\alpha_{2}$ and $\alpha_{3}$ ) we get $\mathscr{W}_{k} \subset \mathscr{W}_{1}$ for all possible $k$ (that is, $k \leqslant g-1$ ).

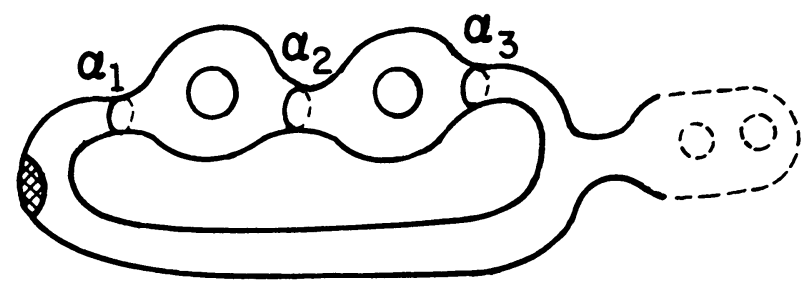

FIGURE 2

IV. We now introduce our main tool, a relation arising from twists on a sphere with four holes (that is, a disc with three holes): see Figure 3. Each $\varepsilon_{i}$ is a curve parallel to a boundary curve and interior to the surface. The twists about the various curves can now be defined in the standard way. Here are our conventions. First, $T_{\alpha}$ means the homeomorphism which affects an arc crossing $\alpha$ by causing it to turn right as it approaches $\alpha$, run once around $\alpha$, and then progress on as before. Second, the order of composition we are using is the functional one: $T_{\beta} T_{\alpha}$ means apply $T_{\alpha}$ first, then $T_{\beta}$. Finally, recall that an equation between twist products means that the two sides are isotopic by an isotopy fixing the boundary. With these conventions then, the following relation holds:

$$
T_{\gamma} T_{\beta} T_{\alpha}=T_{\varepsilon_{1}} T_{\varepsilon_{2}} T_{\varepsilon_{3}} T_{\varepsilon_{4}} .
$$

We can prove the relation by looking at the effect of the map

$$
T_{\gamma} T_{\beta} T_{\alpha} T_{\varepsilon_{1}}^{-1} T_{\varepsilon_{2}}^{-1} T_{\varepsilon_{3}}^{-1} T_{\varepsilon_{4}}^{-1}
$$

on Figure 4 will find that the result can be deformed modulo the boundary back to the original. Since cutting Figure 4 along the three arcs reduces it to a disc, any map which fixes the boundary and arcs must be isotopic to 1; we omit the details. 


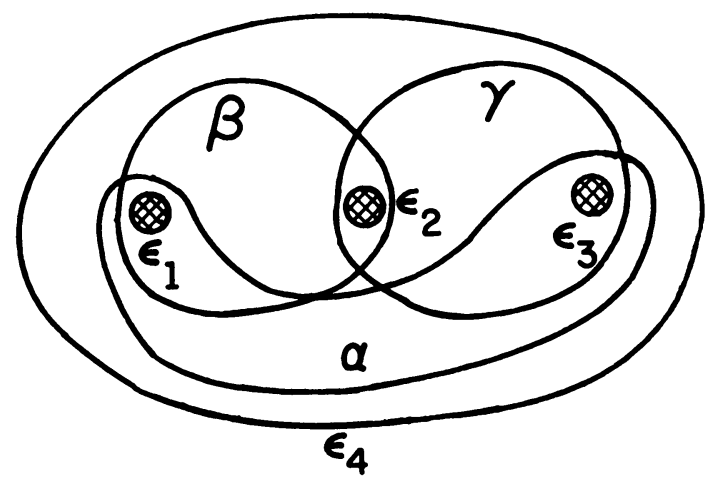

Figure 3

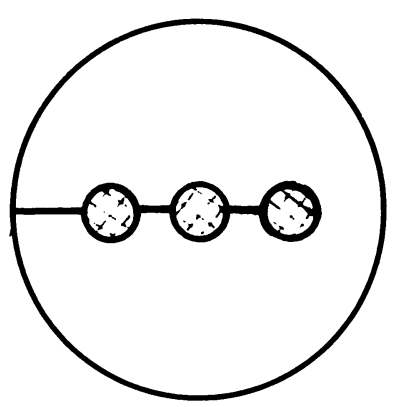

FIGURE 4

We now deform Figure 3 into Figure 5. We will use this lanternlike figure and its relation to derive relations in 9 by the process of glueing various surfaces (with one hole) onto the various holes $\varepsilon_{i}$ of the lantern. Note that since each $\varepsilon_{i}$ is disjoint from all other twist curves in the figure, it commutes with all of them. Hence in the relation

$$
T_{\gamma} T_{\beta} T_{\alpha} T_{\varepsilon_{1}}^{-1} T_{\varepsilon_{2}}^{-1} T_{\varepsilon_{3}}^{-1} T_{\varepsilon_{4}}^{-1}=1,
$$

the $T_{\varepsilon_{i}}$ 's may be placed anywhere and in any order.

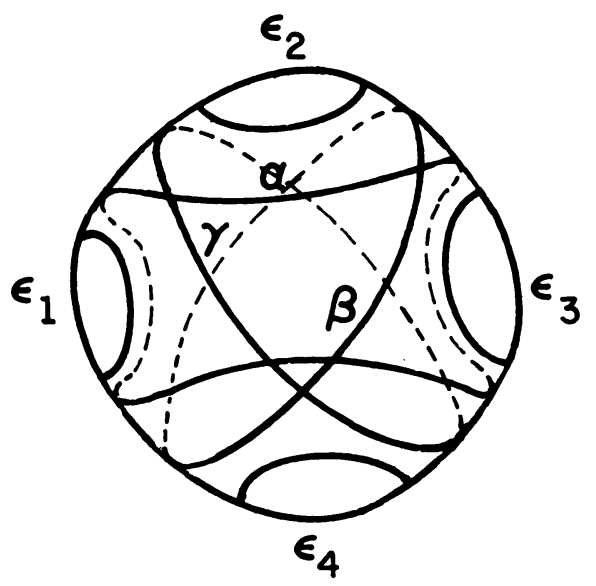

FIGURE 5 
V. To begin with we glue surfaces of genus $1,1, k$ to the curves $\varepsilon_{1}, \varepsilon_{2}, \varepsilon_{3}$ respectively of the lantern: see Figure 6 . We have then:

$$
\begin{aligned}
& g(\alpha)=g(\gamma)=k+1 ; \quad g(\beta)=2, \\
& g\left(\varepsilon_{1}\right)=g\left(\varepsilon_{2}\right)=1, \quad g\left(\varepsilon_{3}\right)=k, \quad g\left(\varepsilon_{4}\right)=k+2 .
\end{aligned}
$$

Here the lantern relation gives us:

$$
T_{\varepsilon_{4}}=T_{\varepsilon_{1}} T_{\varepsilon_{2}} T_{\varepsilon_{3}} T_{\gamma} T_{\beta} T_{\alpha} \in \mathcal{T}_{1} \cdot \mathcal{T}_{2} \cdot \mathcal{T}_{k} \cdot \mathcal{T}_{k+1}
$$

and $T_{e_{4}}$ is a generator of $\mathscr{T}_{k+2}$; thus $\mathcal{T}_{k+2} \subset \mathcal{T}_{1} \cdot \mathcal{T}_{2} \cdot \mathcal{T}_{k} \cdot \mathcal{T}_{k+1}$. By induction, we get $\mathcal{T}_{k} \subset \mathcal{T}_{1} \cdot \mathcal{T}_{2}$ for all $3 \leqslant k \leqslant g$, proving:

THEOREM 1. If $\mathcal{T}$ is the group generated by all twists on BSCC's then $\mathcal{T}=\sigma_{1} \cdot \mathcal{T}_{2}$.
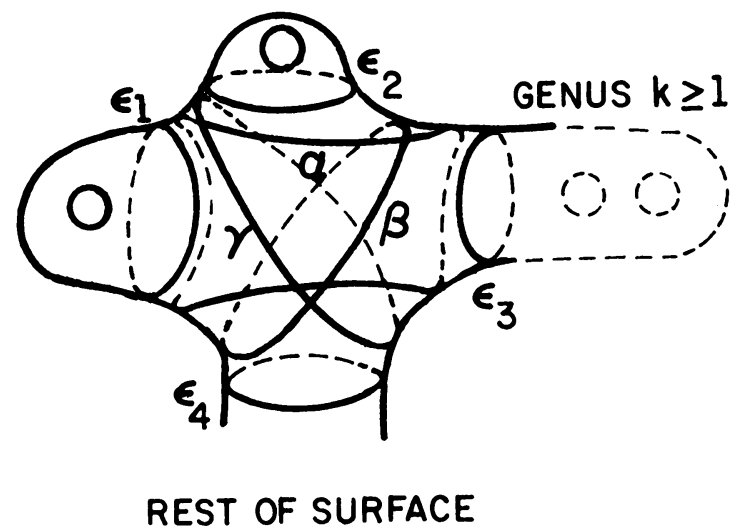

FIGURE 6

Suppose next we delete the genus 1 surface glued to $\varepsilon_{1}$ in Figure 6 , and assume that the resulting figure sits in our surface $M$ so that $\varepsilon_{1} \nsim 0$ (this can be so arranged for $g \geqslant 3$ ). We still have $\alpha \simeq \varepsilon_{4} \simeq \beta \simeq \varepsilon_{1}$, and get:

$$
g\left(\alpha, \varepsilon_{4}\right)=g\left(\beta, \varepsilon_{1}\right)=1, \quad g\left(\varepsilon_{2}\right)=1, \quad g\left(\varepsilon_{3}\right)=k, \quad g(\gamma)=k+1 .
$$

Writing the lantern relation as

$$
T_{\gamma}=T_{\varepsilon_{2}} T_{\varepsilon_{3}}\left(T_{\varepsilon_{4}} T_{\alpha}^{-1}\right)\left(T_{\varepsilon_{1}} T_{\beta}^{-1}\right) \in \mathcal{T}_{1} \cdot \mathcal{T}_{k} \cdot \mathscr{W}_{1},
$$

we see that $\mathscr{T}_{k+1} \subset \mathcal{W}_{1} \cdot \mathcal{T}_{1} \cdot \mathcal{T}_{k}$. In particular, for $k=1$ we get $\mathscr{T}_{2} \subset \mathcal{W}_{1}$. $\mathcal{T}_{1}$. Finally, let us also remove the genus $k$ surface from $\varepsilon_{3}$, and assume (again possible for $g \geqslant 3$ ) that the resulting figure, which now has only one surface of genus 1 glued on (to $\varepsilon_{2}$ ), is imbedded in $M$ so that $\varepsilon_{1}, \varepsilon_{3}$ and $\varepsilon_{4}$ are all nonhomologous to zero. Then we get for the genera of homologous pairs:

$$
g\left(\alpha, \varepsilon_{4}\right)=g\left(\beta, \varepsilon_{1}\right)=g\left(\gamma, \varepsilon_{3}\right)=1, \quad g\left(\varepsilon_{2}\right)=1
$$

The lantern relation now reads:

$$
T_{\varepsilon_{2}}=\left(T_{\gamma} T_{\varepsilon_{3}}^{-1}\right)\left(T_{\beta} T_{\varepsilon_{1}}^{-1}\right)\left(T_{\alpha} T_{\varepsilon_{4}}^{-1}\right) \in \mathcal{W} S_{1}
$$


and hence $\mathscr{T}_{1} \subset \mathcal{W}_{1}$. This shows us then that $\mathscr{T}_{1} \cdot \mathscr{T}_{2} \subset \mathcal{W}_{1}$ and so $\mathscr{T}_{k} \subset \mathscr{W}_{1}$ for all $k$. Using the results of $\S$ II and III, we get finally:

THEOREM 2. $g=W_{1}$.

Note that what we have actually proved is that the group generated by all of the $\mathscr{W}_{k}$ 's and $\mathcal{T}_{k}$ 's is generated by $\mathscr{W}_{1}$ alone. A direct proof that $g$ is the former group would then give us Theorem 2 without the use of Powell's theorem, which is proved indirectly using a nongeometric argument concerning a presentation of the symplectic group $\operatorname{Sp}(g, Z)$.

VI. We are now in a position to show that $[\Re, g]=9$. Consider Figure 7 , let $p$ be the $180^{\circ}$ rotation around the central axis; we get $p(\alpha)=\alpha^{\prime}, p(\beta)=$ $\beta^{\prime}$. Hence $T_{\beta^{\prime}}=p T_{\beta} p^{-1}$ and so $f=T_{\beta} T_{\beta^{\prime}}^{-1}=\left[T_{\beta}, p\right] \in \mathscr{g}$ is a generator of $\mathscr{W}_{1}$. Let $h$ be any homeomorphism such that $h(\beta)=\alpha$, so that $T_{\alpha}=h T_{\beta} h^{-1}$. Then $\bmod [\Re, g]$ we get

$$
\left[T_{\beta}, p\right] \equiv h\left[T_{\beta}, p\right] h^{-1}=\left[h T_{\beta} h^{-1}, h p h^{-1}\right]=\left[T_{\alpha}, p\left[p^{-1}, h\right]\right] .
$$

Notice that the action of $p$ on $H_{1}(M, Z)$ is just negation. Thus its action commutes with any linear map on $H_{1}$, so $\left[p^{-1}, h\right]=1$ on $H_{1}$, i.e., $\left[p^{-1}, h\right]=$ $k$ is in 9 . Now using the standard commutator identity

$$
\left[T_{\alpha}, p k\right]=\left[T_{\alpha}, p\right] \cdot p\left[T_{\alpha}, k\right] p^{-1}
$$

and, noticing that $p\left[T_{\alpha}, k\right] p^{-1} \in[\mathfrak{T}, \mathscr{9}]$, we get

$$
f=\left[T_{\beta}, p\right] \equiv\left[T_{\alpha}, p\right] \bmod [\Re, g] .
$$

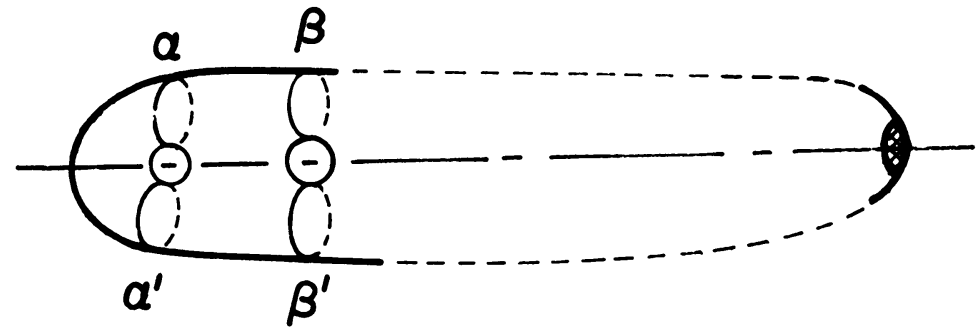

FigURE 7

But $\left[T_{\alpha}, p\right]=T_{\alpha} T_{\alpha^{\prime}}^{-1}=1$ since $\alpha$ and $\alpha^{\prime}$ are isotopic; thus $f \in[\mathscr{\Re}, \mathcal{G}]$. Since $[\mathscr{R}, \mathscr{G}]$ is normal in $\mathscr{N}$, this shows that $\mathscr{W}_{1} \subset[\Re, g]$, that is $\mathscr{G} \subset[\Re, g]$, that is:

THEOREM 3. $[\mathfrak{T}, 9]=9$. 


\section{REFERENCES}

[BC] J. Birman and R. Craggs, The $\mu$-invariant of 3-manifolds and certain structural properties of the group of homeomorphisms of a closed, oriented 2-manifold, Trans. Amer. Math. Soc. 237 (1978), 283-309.

[MKS] W. Magnus, A. Karass and D. Solitar, Combinatorial group theory, Interscience, New York, 1966.

[P] J. Powell, Two theorems on the mapping class group of surfaces, Proc. Amer. Math. Soc. 68 (1978), 347-350.

Jet Propulsion laboratory, California Institute of Technology, 4800 OAk Grove Drve, Pasadena, Californa 91103 